\title{
HALLAZGOS DE LA INVESTIGACIÓN SOBRE LA INSERCIÓN DE LAS TECNOLOGÍAS DE LA INFORMACIÓN Y LA COMUNICACIÓN (TIC) EN LA ENSEÑANZA: LA EXPERIENCIA DE LOS ÚLTIMOS DIEZ AÑOS EN LOS ESTADOS UNIDOS
}

\author{
Flora Eugenia Salas Madriz \\ Docente de la Escuela de Administración Educativa de la \\ Universidad de Costa Rica
}

Recibido 30-VIII-2004

Aceptado 7-IX-2004

\begin{abstract}
Resumen: Debido a que Estados Unidos es el país con mayor trayectoria, inversión estatal y privada $e$ investigación en el campo de las tecnologías de la información y la comunicación (TIC) aplicadas a la educación, su experiencia y reportes de investigación lo convierten en líder mundial en la producción de conocimiento científico sobre este tema. Por esta razón, en este artículo, a partir de una revisión bibliográfica, se hace una sintesis y se reflexiona sobre algunos de los principales hallazgos de la investigación realizada al respecto en ese pais en los últimos diez años, con el objetivo de pormenorizar los principales factores de éxito y fracaso que se ha identificado se encuentran asociados al uso de las TIC como herramientas para mejorar y cambiar la enseñanza y el aprendizaje, así como las tareas aún pendientes para aprovechar y explotar el potencial de estas tecnologías en la educación en general y en particular en el nivel universitario.
\end{abstract}

Palabras clave: Tecnologías de la información y la comunicación aplicadas a la educación, tecnologías de la información y la comunicación en educación superior, educación superior, cambio en educación superior.

\section{Introducción}

Después de dos décadas de grandes inversiones a escala global en infraestructura, equipo y programas para la incorporación de las tecnologías de la información y la comunicación (TIC) en todos los niveles educativos, los resultados alcanzados en el presente no muestran un avance substancial ni un cambio significativo. Por el contrario, la crisis en la calidad de la educación fue generalizada en la última década y se manifiesta hasta hoy en el bajo rendimiento académico en primaria y secundaria, y en el aumento de la deserción y la repitencia, particularmente en los países del Tercer Mundo (Kliksberg, 2004). Estos fenómenos son importantes para la educación superior, ya que la investigación muestra que el deterioro en los resultados de la educación primaria y secundaria incide en la calidad, pertinencia y equidad de la formación universitaria, pues afecta de manera directa las características y calidades de la población que accede a ella (Research Institute for Higher Education of Hiroshima University, 2000). 


\begin{abstract}
The United States is the world leader in the production of knowledge in the field of information and communication technology (ICT) because of its long-standing tradition, public and private investment, experience and research projects applied to education. This article provides a synthesis on some of the main findings in research done in this country for the last ten years. The purpose of this study is to highlight the most important factors of success and failure that have been identified in relation with the use of ICT as a tool to improve and change teaching and learning. The pending tasks to take advantage of the potential of these technologies for education in general and for higher education in particular are also discussed.
\end{abstract}

Key words: Higher education, information and communication technologies, change in higher education.
En términos generales, como consecuencia de la pérdida de la calidad educativa en los niveles primario y secundario, las carreras universitarias de ciencia y tecnología reciben en la actualidad pocos estudiantes y la mayoría de jóvenes que ingresan a la universidad carece de los conocimientos, habilidades y destrezas necesarios para enfrentar procesos de enseñanza de orden superior. En la región latinoamericana, el poco dominio de la lengua materna, las matemáticas y las ciencias, y el desinterés de la juventud por la educación formal se suman a una pobreza creciente, en una economía globalizada donde quienes no tienen una sólida formación académica y no están alfabetizados computacionalmente están condenados a bajos salarios, al subempleo $y$, en el peor de los casos, al desempleo (Kliksberg, 2004).

Debido al "desencanto" producido por la baja incidencia de las TIC en el mejoramiento de la calidad y el cambio de la enseñanza, el apoyo a la investigación en esta área en los Estados Unidos se ha incrementado en los últimos diez años. El principal propósito de muchas de las investigaciones realizadas hasta ahora en esa nación, ha sido determinar éxitos y fracasos en distintas experiencias de enseñanza y aprendizaje mediadas por tecnología en diferentes contextos alrededor del mundo, para descubrir cuáles acciones realmente permiten que estas herramientas contribuyan al "despegue" de la educación necesaria en la Era del Conocimiento y la Globalización.

A continuación, con base en una revisión bibliográfica, presentamos algunos de los principales hallazgos de la investigación sobre la incorporación en la enseñanza de las TIC en los últimos diez años en los Estados Unidos, ya que es el país donde se ha acumulado más experiencia en este campo. Por esta razón, este artículo tiene como propósito ofrecer un panorama general, con base en la literatura consultada, de las principales evidencias empíricas sobre el éxito, el fracaso y las tareas aún pendientes para realmente aprovechar el potencial 
para el cambio y el mejoramiento de la calidad de la enseñanza que ofrecen estas tecnologías, bajo el supuesto de que podemos aprender de estas lecciones y reflexionar sobre el rumbo que hemos dado a la inserción en la enseñanza de estas herramientas en la educación de nuestro país.

\section{La fase ingenua: la tesis de que las TIC pueden por sí mismas cambiar y mejorar la enseñanza y el aprendizaje}

A mediados de la década de los 80 , con la introducción de las computadoras en los Estados Unidos en la enseñanza básica y en secundaria, y como resultado de las investigaciones, experiencias y libros de Seymourt Papert, particularmente su obra The children's machin. Rethinking school in the age of the computer, donde se hace una entusiasta apuesta a los computadores como herramientas capaces de cambiar la educación de manera radical y para siempre, rápidamente creció la expectativa general sobre estos dispositivos bajo el supuesto de que su sola presencia en las instituciones educativas bastaba para mejorar la enseñanza y el aprendizaje y dar un salto cualitativo sin precedentes en la historia reciente de la educación. Como consecuencia, a finales de los 80, muchos países auspiciados por organismos financieros internacionales, agencias de desarrollo gubernamentales y no gubernamentales, nacionales y extranjeras, y por la empresa privada, empezaron a equipar sus escuelas con laboratorios de cómputo sin prever que la puesta en escena de estas máquinas equivalía a abrir una especie de "Caja de Pandora" en el sistema educativo tradicional.

En la fase ingenua, la palabra "tecnología” se trató en los niveles político y administrativo de la educación como una especie de "mantra" que significaba progreso y modernización y, por ende, cambio, calidad y mejores resultados (Novek, 1999). Sin embargo, las computadoras entraron a las aulas sin un proceso de planificación adecuado que garantizara la apropiada utilización y la sostenibilidad técnica, pedagógica y administrativa de los sistemas, razón por la cual rápidamente los equipos se deterioraron, las expectativas de docentes, administradores y estudiantes decayeron y, con el tiempo, los cajones y monitores se amontonaron en bodegas, malográndose así un esfuerzo económico poco frecuente en la historia reciente de la educación.

Las enseñanzas de esta fase "ingenua" han sido más que reveladoras, ya que, en términos generales, la investigación ha demostrado que la puesta en práctica de una innovación educativa en la que no se haga un esfuerzo previo de planificación y administración, no se involucre a las personas que efectivamente van a utilizarla, y no se tomen en cuenta las necesidades e intereses de los usuarios, sólo produce resistencia al cambio y el endurecimiento de las posiciones tradicionales (Lee, 2001). La razón de esta resistencia es consecuencia de la falta de atención a las actitudes, percepciones, e intereses de las personas respecto de la innovación. Por esta razón, hoy sabemos que conocer estos aspectos es fundamental para que el proceso de cambio y la introducción de nuevos dispositivos de enseñanza e innovación educativa sean exitosos.

En términos generales, con base en el metaanálisis de la investigación en el área hecho por The Association for Educational Communications and Technology de los Estados Unidos, en ese país en la década de los 90, se determinó que los principales diez obstáculos para que las TIC sean empleadas adecuadamente en la enseñanza son los siguientes (Mehlinger \& Powers, 2000):

1. Falta de visión: Si entendemos "la visión" como la concepción de lo que es posible, no ha habido una visión apropiada del papel de la tecnología en educación, capaz 
de lograr que ésta mejore la enseñanza y el aprendizaje.

2. Ausencia de planificación: la falta de visión conlleva a la falta de planeamiento, ya que no hay planes bien articulados para capacitar al personal docente, ni para modificar el currículo o evaluar resultados.

3. Apoyo insuficiente a la investigación en el campo: La investigación ha demostrado que, en la mayoría de los casos estudiados, los docentes realmente no sabían como utilizar estas tecnologías en la enseñanza y el aprendizaje y no contaban con tiempo ni las condiciones necesarias para experimentar con ellas y documentar estas experiencias. Dar espacios para la capacitación y la experimentación educativa con estas tecnologías es fundamental para que el profesorado realmente tome el riesgo de implementar de forma innovadora estas herramientas en su trabajo de aula.

4. Infraestructura limitada, inadecuada o ambas: Si se desconocen las necesidades e intereses de los usuarios, la infraestructura informática, por lo general, se diseña e implementa con base en criterios técnicos y económicos, por lo que sus resultados finales son realmente pobres, nulos o, incluso, negativos.

5. Limitado acceso al equipo y a otras tecnologías: por las razones expuestas en el punto anterior, el profesorado encuentra que, si bien hay laboratorios y equipo disponible, el tiempo y recursos para su uso en clase es limitado, lo que lejos de ayudar y facilitar las tareas de aula se convierte en un problema imposible de resolver; como consecuencia, en la mayoría de los casos, la mejor opción para el docente es descontinuar su uso y regresar a las prácticas tradicionales.

6. Falta de incentivos: El profesorado, lejos de encontrar en la tecnología un apoyo a su trabajo, lo que consigue son problemas nuevos para los que no tiene respuesta o solución adecuada de parte de la administración. Esto provoca frustración e incremento de trabajo con resultados académicos mínimos, nulos e, incluso, negativos.

7. Falta de soporte técnico: Uno de los problemas críticos en cuanto al uso de la tecnología es el adecuado funcionamiento de los equipos y el software. Si el profesorado no tiene la garantía que va a contar con ayuda oportuna en el momento que se presente un problema, probablemente después de la primera mala experiencia desista de utilizarlos en sus clases y cursos.

8. Desarrollo profesional insuficiente del profesorado: Es común que el profesorado no tenga el conocimiento técnico y pedagógico necesario para utilizar en forma adecuada las TIC en su trabajo de aula. Si esta formación no se propicia por parte de las autoridades educativas, difícilmente los docentes tendrán la motivación y el interés necesarios para explorar el uso de estas herramientas en sus cursos y actividades académicas. El desarrollo profesional del profesorado es una responsabilidad ineludible de quienes administran la educación, particularmente en lo que respecta al uso de las TIC en el aula, dada la complejidad que encierran estos dispositivos, tanto en el nivel educativo como en el técnico.

9. Requerimientos de capacitación tipo "just-in-time": debido a que cada nivel educativo y cada disciplina o área de enseñanza tiene sus propias características pedagógicas, es necesario el diseño de planes de capacitación docente coherentes con las características, necesidades e intereses de cada sector y nivel. Además, se debe monitorear en forma permanente la evolución y desarrollo de dichas necesidades e 
intereses, a fin de poder responder en forma oportuna a los requerimientos de formación que se vayan generando a lo largo del tiempo.

10. Falta de dinero: Finalmente, la falta de recursos financieros y humanos para atender todos los requerimientos administrativos, de infraestructura, equipo y formación del profesorado asociados al uso adecuado de las TIC en la enseñanza y el aprendizaje, se presenta hoy como uno de los principales problemas de la educación contemporánea.

\section{La nueva perspectiva: de la ingenuidad a la complejidad}

En1996, la Association for Educational Communications and Technology de los Estados Unidos, definió tecnología educativa como: “... a complex, integrated process involving people, procedures, ideas, devices, and organization for analyzing problems, and devising, implementing, evaluating, and managing solutions to these problems, in situations in which learning is purposive and controlled" (Mehlinger \& Powers, 2000, p. 10). Como se puede observar, en esta nueva definición se parte de una concepción compleja de la tecnología educativa, donde las personas, las ideas, los procedimientos, la organización y los dispositivos son igualmente relevantes para resolver problemas en situaciones concretas de enseñanza y aprendizaje, lo que ha permitido un cambio radical de enfoque del potencial que tienen las TIC para contribuir a un cambio significativo en la educación.

A partir de esta nueva visión del uso de las TIC en educación se considera que estas herramientas pueden hacer la enseñanza y el aprendizaje más efectivos, accesibles e interesantes, siempre y cuando la organización educativa se prepare para que el profesorado desarrolle las competencias necesarias para utilizarlas de modo que pueda aprovechar sus ventajas y potencialidades pedagógicas. Desde esta nueva perspectiva, la organización y la administración educativas cobran particular relevancia, ya que, independientemente del nivel educativo de que se trate, resulta indispensable un proyecto institucional definido y adecuadamente gestionado de inserción de estas tecnologías en la enseñanza para que haya innovación y cambio. Por esta razón, la organización educativa debe crear las condiciones necesarias para certificar la solución de problemas de equipo y mantenimiento, reducir al mínimo las limitaciones de acceso de estudiantes y profesores(as) a equipo y a soporte técnico, crear incentivos para el uso de las TIC en la enseñanza y desarrollar espacios de comunicación entre docentes, estudiantes y administradores. Por estas razones, es indispensable identificar intereses, necesidades y expectativas del profesorado para el diseño de planes integrales de incorporación de las TIC en la docencia.

Como se verá a continuación, el enfoque de la inserción de las TIC en función de las personas, es decir, del profesorado y del estudiantado, tanto en la educación primaria y secundaria como en la superior, ha mostrado ser el más adecuado y el que mayores éxitos reporta a la fecha.

\section{Las TIC al servicio de profesores, profesoras y estudiantes}

La investigación en el campo de la inserción de las TIC en el trabajo de aula, muestra que en todos los casos “... los profesores se convierten siempre en los mediadores y agentes básicos de las innovaciones en la Universidad (siempre como aplicadores y, con frecuencia, como instigadores y planificadores de los cambios)" (Zabalza, 2002, p. 14). El profesorado ha resultado ser uno de los principales agentes al introducir innovaciones pedagógicas y didácticas (Bates, 2001; Menereo \& Pozo, 2003; Novek, 1996, 1999; Shelley, Wepner 
\& Nancy Ziomek, \& Liqing, 2003; Zabalza, 2002, 2003, Research Institute for Higher Education of Hiroshima University, 2000). Asimismo, el estudiantado universitario, dado su nivel de madurez e intereses, alto bagaje educativo, opciones profesionales definidas y, condiciones sociales y culturales, constituye una clientela educativa claramente diferenciada, con capacidad de determinar, al menos en parte, el trabajo y proceso de enseñanza y aprendizaje (Zabalza, 2002).

Ambos actores, profesores y estudiantes, entran en una íntima relación en el aula. El espacio educativo privado que se teje entre docentes y estudiantes en cada curso es único e irrepetible y supone relaciones sociales y desarrollo personal. Ningún participante de este proceso sale intacto de la experiencia de aula. Para cada uno esta experiencia marca, pauta, determina, encausa y orienta las expectativas educativas y personales futuras. La convivencia en el aula, las prácticas, acciones, niveles de comunicación y procesos que se viven en su interior, hacen posible la enseñanza y el aprendizaje. Ahora bien, como campo de interacción social, la comunicación juega un papel fundamental en la calidad de las experiencias en el aula y, por ende, en la enseñanza y el aprendizaje. Entre mayores y mejores sean las habilidades y posibilidades comunicativas de docentes y estudiantes, mejores serán sus experiencias y aprendizajes.

En un estudio realizado en los Estados Unidos por Young (1999), sobre docentes universitarios efectivos, se logró determinar que los estudiantes valoraron como buenos profesores aquellos que se inscribían dentro de los siguientes criterios:

1. El curso que imparte resulta valioso y útil para la formación del estudiante.

2. Motiva al estudiante a dar lo mejor de sí.

3. Propicia una atmósfera de aprendizaje confortable.
4. Hace una adecuada organización del curso.

5. Tiene una comunicación efectiva.

6. Se preocupa por el aprendizaje de sus estudiantes.

Como se puede observar, sólo el primer aspecto de los criterios de evaluación de los estudiantes sobre la efectividad del profesor o profesora tiene que ver con los contenidos del curso, lo otros cinco criterios apuntan a rasgos de personalidad asociados a habilidades sociales y comunicativas. Con base en estos hallazgos, resulta evidente que dichas habilidades son un aspecto crítico en el éxito o fracaso de los procesos de enseñanza y aprendizaje. Por lo anterior, se puede concluir que si se logran mejorar las condiciones y posibilidades de comunicación dentro del aula, es muy probable que ello incida en un mejoramiento general de los aprendizajes. Las TIC tienen el potencial de elevar la capacidad comunicativa del profesorado. No es lo mismo la voz, la imagen o el texto aislados, que la combinación de estos elementos. Imagen, movimiento, color, sonido e información presentada en distintos formatos, se conjugan para enriquecer la experiencia sensible de estudiantes y docentes, permitiéndoles elaboraciones cognitivas significativas.

Gracias a la investigación realizada en el campo de la psicología cognitiva y psicogenética en el siglo pasado, hoy sabemos que el aprendizaje significativo “... es aquel que conduce a la creación de estructuras de conocimiento mediante la relación sustantiva entre la nueva información y las ideas previas de los estudiantes" (Díaz-Barriga \& Hernández, 2001, p. 39). El sentido de lo visto, escuchado y pensado se articula a través de procesos mentales más complejos, ya que involucra de manera específica la vista y el oído, sentidos capaces de evocar recuerdos que incorporan sensaciones, experiencias anteriores, pensamientos e ideas previos, que intervienen como detonadores de aprendizajes significativos. Percepción, memoria y pensamiento se encuentran 
indisolublemente ligados como procesos de índole biológica que permiten la adaptación y reacción de los seres vivos al entorno (Banyard, Cassells, Green, Hartland, Hayes \& Reddy, 1995).

También, gracias a estas investigaciones hoy sabemos que existe un importante componente afectivo en la construcción del conocimiento, por lo que no podemos obviar que en toda experiencia educativa subyace una experiencia emocional que pauta el interés y motivación del aprendiz y del educador o educadora (Piaget, 2001). Experiencias de enseñanza y aprendizaje poco gratificantes estética, intelectual y afectivamente, inevitablemente son malas experiencias, que trataremos de no repetir y de olvidar lo antes posible. Como bien mostró Piaget:

No hay mecanismo cognitivo sin elementos afectivos [y] tampoco hay un estado afectivo puro sin elementos cognitivos. [Si bien hay que distinguir] entre las funciones cognitivas (que van desde la percepción y las funciones sensorio-motrices hasta la inteligencia abstracta, incluidas las operaciones formales, y las funciones afectivas. Distinguimos estas dos funciones porque nos parecen de naturaleza diferente, pero en el comportamiento concreto del individuo son indisociables. Es imposible encontrar comportamientos que denoten únicamente afectividad, sin elementos cognitivos y viceversa. (Piaget, 2001, p. 19).

Los(as) educadores(as) podemos utilizar a nuestro favor el componente afectivo intrínseco a los procesos cognitivos $-\mathrm{y}$ de hecho lo hacemos-, que, como señalamos anteriormente, obedece a la lógica psicobiológica de los seres humanos y constituye la plataforma de nuestra psique y nuestra sociabilidad. El enlace con el entorno entre el organismo y la mente lo producen los sentidos y entre más sentidos intervengan, más intensa será la experiencia y mayor cantidad de información estará disponible para que el cerebro la procese en función de códigos y sentido, dándole al individuo el conocimiento necesario para tomar las mejores decisiones, en función de su bagaje y circunstancias (García, 2000; Piaget, 2001; Rosas \& Sebastián, 2001; Vygotski,
1995). Percepción, lenguaje, pensamiento y afectividad son componentes de un mismo proceso en el que se hace posible el aprendizaje y, por ende, la capacidad específica de nuestra especie de adaptar el entorno a sus necesidades.

Si bien las tecnologías de la información y la comunicación potencian la capacidad comunicativa humana, es un hecho que deben ser adecuadamente utilizadas para que esto ocurra, ya que su mal empleo puede producir el efecto contrario o, en el mejor de los casos, no producir ningún efecto. Esto es lo que ha mostrado la investigación en esta área, ya que el sonido, el color, la forma y el movimiento, así como la cantidad y calidad de información, deben ser apropiadamente articulados para elevar el aprovechamiento del potencial de aprendizaje en el aula. Si estas tecnologías se emplean en forma lineal, al estilo tradicional de los textos escritos, probablemente su impacto sea menor que si se utilizan como multimedios, definidos como la:

Integración de dos o más medios de comunicación que pueden ser controlados o manipulados por el usuario mediante el ordenador [...] vídeo, texto, gráficos, audio y animación controlada con ordenador [...] combinación de hardware, software y tecnologías de almacenamiento incorporadas para proveer un ambiente de información multisensorial. (Cabero, Martínez \& Salinas, 1999, p. 366).

También, y de forma aún más compleja, las TIC se pueden emplear como hipermedios, definidos como:

Un estilo de sistema que gestiona la información [...] en una red de nodos multimedia interconectados por enlaces. Un hipermedia sería un medio multi-expresión. El cuerpo del material a incluir puede ser texto, gráficos estáticos y animados, voz, sonido y música. [...] Un buen diseño del sistema debe permitir a los alumnos enlazar información, crear sus propias rutas a través del material, realizar anotaciones y literalmente construir redes de información. (Cabero, Martínez \& Salinas, 1999, p. 366).

Como se puede observar, el reto educativo de nuestro tiempo es sacar ventaja y explotar inteligentemente el potencial de 
las TIC, lo que nos permitiría dar el salto de la pedagogía y las teorías del aprendizaje regidas por la lógica del discurso impreso y lineal -que privilegian la memoria (Burke \& Ornstein, 1997; McLuhan, 1962; Olson, 1999)-, hacia pedagogías no lineales y teorías del aprendizaje regidas por lógicas discursivas combinadas articuladas en redes -que privilegian el pensamiento significativo y complejo (Majò \& Marquèz, 2000; Rodríguez, 2002; Vizcarro \& León, 1998). De hecho, ya McLuhan señaló de manera extraordinaria en sus libros The Gutenberg Galaxy, publicado en 1962 y Understanding Media. The Extensions of Man, publicado en 1964, como el medio en el que representamos el conocimiento y el pensamiento determina, a su vez, la forma como conocemos y pensamos el mundo y a nosotros(as) mismos(as).

En la misma línea de McLuhan, Burke \& Ornstein (1997), Olson (1999) y Castells (2001), sostienen la tesis de que la cultura impresa y la cultura digital, permiten explicar la forma como conocemos $\mathrm{y}$, por ende, la forma como enseñamos $\mathrm{y}$ aprendemos en cada uno de esos contextos culturales. Hoy, es un hecho que estamos pasando de la cultura impresa a la digital, razón por la cual no sólo enfrentamos el impacto de las tecnologías digitales en todas las esferas de la vida social, económica y cultural, sino también estamos en medio de una transformación epistemológica radical que está cambiando la manera como conocemos, pensamos y sentimos al mundo y a nosotros mismos. Esto, indudablemente tiene profundas implicaciones para la educación.

Ahora bien, si asumimos que el acto educativo es en esencia un acto de comunicación, cualquier medio que contribuya a mejorarla y hacerla más efectiva irá, a su vez, a contribuir al mejoramiento de la enseñanza y el aprendizaje. Además, se ha demostrado que las personas que participan y aprovechan estos recursos deben saber emplearlos adecuadamente en los niveles técnico y sémico, sentirse cómodas con su uso, y ser conscientes de los beneficios que les proporcionan; de lo contrario, el medio no será de provecho para ninguna de las partes.

\section{Las TIC en el escenario global: la nueva economía y la formación profesional}

El cambio de la Sociedad Industrial a la Sociedad de la Información es el resultado de una profunda transformación económico-social, producida por tres grandes revoluciones científico-tecnológicas: la microelectrónica, la informática y las telecomunicaciones. Gracias a estas innovaciones, la humanidad se colocó en posición de "cambiar de época": de la Era Industrial a la Era Internet (Castells, 2001); y, obviamente, ello tiene implicaciones para la educación. Por esta razón, si hoy es incuestionable la necesidad de elevar la calidad de la enseñanza, también lo es ajustarla a las nuevas demandas y requerimientos de la sociedad contemporánea. La revolución producida por las TIC no sólo ha cambiado la cultura y los medios de comunicación masivos, también ha tenido un impacto significativo e irreversible en el mundo del trabajo. Este hecho concierne especialmente a la educación superior, ya que en nuestro tiempo se necesitan personas alfabetizadas en el uso de estas tecnologías y educadas a lo largo de la vida.

Los entornos laborales y la creciente complejidad de la actividad científica exigen profesionales que sepan utilizar y aprovechar las TIC. De hecho, los puestos de trabajo de hoy se caracterizan por una incorporación progresiva de estas tecnologías en todos los niveles: administrativo, productivo, técnico y científico. Profesionales poco alfabetizados en esta área serán mucho más vulnerables a los cambios y nuevos requerimientos del mundo del trabajo y, por ende, una educación superior que no provea esta formación a sus estudiantes, les estará restando 
oportunidades de desarrollo profesional y laboral. Por esta razón, el reto de la educación contemporánea no es sólo sacar el máximo provecho de las TIC para elevar la calidad educativa, sino también brindar a la población estudiantil la oportunidad de obtener una adecuada alfabetización en su uso para el desarrollo académico, personal, laboral y profesional. Como se expuso anteriormente, “... la introducción de las nuevas tecnologías en las aulas y los consiguientes cambios en los métodos de enseñanza deben ser contemplados como un proceso de mejora, que supone la necesidad de formar e informar adecuadamente a sus futuros usuarios" (Majò \& Marquès, 2000, p. 17).

El empleo de las TIC es un fenómeno que toca a la sociedad en todas sus dimensiones y rebasa con mucho los aspectos específicamente técnicos y tecnológicos, pues es un acontecimiento que tiene implicaciones en los niveles político, cultural, económico y social (Cabero, 2001). Como se mencionó, estas tecnologías también han revolucionado el mundo del trabajo, al trastocar la lógica de la formación, la oferta y la demanda de profesionales, debido a que, como señalan Prieto y otros (citados en: Cabero, 2001, p. 43), el impacto de las TIC en el mercado laboral se refleja en las siguientes dimensiones:

a. Cambio en la actividad laboral y en el puesto del trabajo, ya que la necesidad de adquirir nuevos conocimientos y formación de manera continua se vuelven parte de la actividad laboral misma.

b. En la estructura del trabajo, debido a que se desarrollan nuevos arreglos y modalidades laborales en función de la creciente relación entre personas, máquinas y productividad.

c. Cambio en las actividades y tareas, pues se eliminan las actividades tradicionales, jerarquizadas y rígidas para ser reemplazadas por nuevas estrategias de trabajo más horizontales, flexibles, autónomas, cooperativas y creativas. d. Nuevas características del puesto de trabajo, ya que se han modificado sustancialmente las relaciones de autoridad, el grado de control del trabajador sobre el producto final $\mathrm{y}$, la calificación y valoración del puesto en función del grado de profesionalización y dominio técnico.

e. Desarrollo de habilidades y destrezas superiores, debido a que se incrementa la necesidad de habilidades y destrezas nuevas por parte de los trabajadores para continuar desempeñándose adecuadamente en sus puestos de trabajo.

f. Nuevas necesidades psicosociales en el trabajo, ya que se requiere de personas con un alto grado de autonomía y creatividad, capaces de tolerar niveles elevados de presión, entornos laborales cambiantes, actividades de trabajo en equipo y que, además, sean tolerantes a la diferencia, la diversidad y la frustración.

g. Pérdida de estabilidad en el puesto de trabajo y en el desempeño de roles, debido a que es probable que las personas tengan que enfrentar a lo largo de sus vidas cambios en su entorno laboral, tanto dentro de sus empresas u organizaciones, como al trasladarse hacia otras, debido a la naturaleza altamente cambiante de los procesos productivos postindustriales.

h. En la promoción, reciclaje y desarrollo de la carrera profesional, como resultado del acelerado avance del conocimiento, la aparición de nuevos puestos y la desaparición de otros.

i. En el entorno social del trabajo y las relaciones interpersonales, consecuencia de la mediación de las TIC, y, por ende, de la reducción del contacto y trato personales.

j. En la productividad, la capacidad de influencia y la participación en la toma de decisiones de 
los(as) trabajadores(as), producto de actividades de trabajo más complejo, especializado e interdisciplinario.

Como hemos señalado anteriormente, la incorporación de las TIC en educación superior no es sólo un asunto pedagógico, de gestión e infraestructura, sino que implica también repensar los planes de estudio, los tiempos de graduación, las necesidades del mercado laboral y, las estrategias de actualización y revisión de los mismos, a fin de garantizar su pertinencia y su calidad.

\section{Ventajas adicionales de la incorporación de las TIC en educación superior: tareas pendientes}

A continuación presentamos algunas de las tareas más importantes aún pendientes en nuestro tiempo, según los hallazgos de la investigación en el campo de la incorporación de las TIC en la administración, la docencia, la acción social y la investigación universitarias, (Research Institute for Higher Education of Hiroshima University, 2000). También, como veremos, estas tareas están pendientes en las instituciones de educación superior costarricenses.

\subsection{La creciente demanda por educación continua: actualización y reciclaje profesional}

Entre las nuevas demandas educativas más importantes, y que requiere respuesta en el corto plazo, está un nuevo grupo de estudiantes, compuesto por adultos graduados, ubicados laboralmente, que necesitan formación y reciclaje profesional para elevar su productividad y competitividad laboral. A estos procesos de formación se les llama en la actualidad "educación continua", término que procede del inglés long-life learning. Estos nuevos estudiantes universitarios son radicalmente diferentes a los tradicionales, ya que, debido a que se encuentran ubicados laboralmente, sólo pueden dedicarse a estudiar a tiempo parcial, y, por lo general, a él pertenecen minorías, mujeres, emigrantes y adultos medios. Esta población creciente es consecuencia, a su vez, de los profundos cambios demográficos y de las transformaciones y exigencias del mercado laboral de nuestro tiempo (Research Institute for Higher Education of Hiroshima University, 2000).

Las TIC, dada su capacidad para superar las barreras espacio-temporales, permiten el diseño de programas de educación continua bimodales y a distancia, que facilitan el acceso a educación y formación a personas que tienen limitaciones de tiempo y carecen de las condiciones para participar en sistemas de formación presenciales. En esta área, las TIC ofrecen una alternativa que no puede ser desestimada por los administradores de la educación superior.

Los nuevos escenarios laborales demandan personal altamente formado y flexible, con capacidad y posibilidades de reciclaje, recualificación y conversión laboral, por lo que para expertos en el campo,

El hecho más significativo es que muchos de los trabajos nuevos que se crean requieren un grado de capacitación mucho mayor que los trabajos a los que sustituyen, especialmente en las industrias de gestión y producción. Las personas conservarán su empleo sólo si adquieren una formación de mayor nivel; incluso, para la mayoría de los trabajos escasamente remunerados y que normalmente exigen un nivel de capacitación bajo, serán necesarias la formación o el reciclaje, sobre todo en las destrezas básicas, sólo para poder conservar su empleo. (Bates, 2001, p. 29).

La población laboralmente activa de hoy requiere habilidades, destrezas y conocimientos nuevos en forma constante, y estar dispuesta a reciclarse en el momento apropiado. Aunado a esto, en la formación universitaria se deben fortalecer ciertas habilidades y destrezas que constituyen la base de la funcionalidad laboral en nuestro tiempo: 
1. Destrezas de comunicación: lectura, escritura, comunicación oral y capacidad para escuchar.

2. Capacidad para aprender de forma independiente.

3. Destrezas sociales: actitudes responsables, éticas y constructivas.

4. Capacidad para el trabajo en equipo.

5. Capacidad para adaptarse a circunstancias cambiantes.

6. Habilidades y destrezas de razonamiento, resolución de problemas, pensamiento lógico y numérico.

7. Capacidad para buscar, encontrar y procesar información. (Bates, 2001, p. 30).

Las TIC pueden ser un importante medio para el desarrollo y fortalecimiento de estas habilidades y destrezas en todos los niveles educativos y, particularmente, en educación superior.

Como se puede observar, las crecientes demandas sociales, económicas y laborales por reciclaje, recualificación y conversión laboral, hacen que la educación continua sea una de las nuevas funciones de la educación superior contemporánea.

\subsection{Vinculación con la sociedad}

Una de las funciones históricas de la educación superior es dar acceso a la sociedad a las distintas formas de la cultura nacional e internacional, con el propósito de abrir oportunidades para el aprovechamiento de los logros artísticos, científicos y tecnológicos de la humanidad. Si bien hoy muchas universidades públicas, extranjeras y costarricenses, cuentan con medios de comunicación y transmisión de la cultura como canales de televisión, emisoras de radio, periódicos, editoriales, teatros, museos y revistas, en términos generales, éstos siguen teniendo una baja audiencia y aún no se explora el potencial de promoción y transmisión cultural de la Internet.

Continuar con esta función y aprovechar las TIC para elevar el interés y el acceso de la población a la cultura, la ciencia y el arte, es una tarea pendiente.

\subsection{Vinculación con los sectores productivos}

El acelerado desarrollo de innovaciones científicas y tecnológicas hace que en la actualidad el conocimiento experimente una rápida obsolescencia. Debido a esto, el cambio y transformación de los planes de estudio, así como las áreas críticas de investigación y desarrollo profesional se deben monitorear en forma permanente, con el propósito de que la formación universitaria realmente responda a las necesidades y requerimientos de los sectores productivos.

Es un hecho que hoy muchas de las universidades en los niveles mundial y local no forman profesionales dentro de los perfiles requeridos por la economía contemporánea. Nuevamente, en este escenario las TIC ofrecen posibilidades ilimitadas para conectar las instituciones de educación superior con los sectores productivos. La Internet permite enlazar a empresas, industria y organizaciones con investigadores, docentes y estudiantes universitarios, para construir redes de investigación y desarrollo especializado y de alto nivel.

Una red que una a los sectores productivos con las instituciones de educación superior no sólo es factible en nuestros días, sino que, sin duda, produciría beneficios incalculables para ambos sectores, pues mejoraría la formación y utilización de los recursos humanos, físicos y financieros para la generación, transferencia y desarrollo de ciencia y tecnología, con el consiguiente impacto en la productividad, el desarrollo económico y el empleo.

Cerrar la brecha entre la formación profesional y los requerimientos de los sectores productivos, es una tarea pendiente. 


\subsection{Eficiencia y eficacia administra- tivas: una gestión orientada al cliente}

La administración universitaria en muchos lugares del mundo -y la costarricense no es la excepción-, aún se rige por la teoría administrativa de corte burocrático, en la que el cliente no es el eje que articula el quehacer de la organización, sino alguien que, de algún modo, "la padece". Los sistemas de matrícula, los trámites de pago, el acceso a los servicios, y, en general, todos los trámites asociados a ser estudiante o docente en estas instituciones, aún carece de una orientación real y efectiva al cliente.

Las TIC, debido a su capacidad para almacenar, tramitar y procesar información, son una herramienta de incalculable valor para realizar trámites administrativos. Si se cuenta con sistemas de información y bases de datos diseñadas para dar servicio al cliente, es probable que la calidad de los servicios y la satisfacción de los usuarios mejoren, contribuyendo ello a elevar la calidad de vida de la población estudiantil, docente, administrativa y del público en general. Pero, esto no es todo, la eficiencia en la entrega de los servicios se traduce en una reducción de costos colaterales, que libera recursos para desarrollar sistemas administrativos de mejora continua. No hay nada más caro que la ineficiencia administrativa y, lo que es peor, no hay nada menos inteligente en términos administrativos que obligar a la organización a ser ineficiente por falta de recursos y por el mal diseño de procedimientos.

Poner la administración universitaria al servicio del cliente interno y externo a la institución, es una tarea pendiente.

\section{Reflexión final}

Como hemos podido observar, los primeros pasos en la incorporación de las TIC en la enseñanza en general y, en particular, en educación superior, constituyen un largo proceso de ensayos y errores de los cuales la investigación ha permitido extraer importantes aprendizajes, tanto en el nivel pedagógico como administrativo.

La necesidad de formación y profesionalización continua del profesorado, la alfabetización computacional, el acceso a equipo y software adecuado para estudiantes y profesores, sistemas robustos de mantenimiento de equipo, planificación y previsión administrativa, son condiciones necesarias y el punto de partida de una apropiada incorporación de estas tecnologías que permita el aprovechamiento de su potencial para el cambio de la educación superior contemporánea.

Ahora bien, este es solo el principio, ya que aún quedan tareas pendientes en lo que respecta al aprovechamiento de estas tecnologías para poner la formación universitaria a la altura de los tiempos. Emplear las TIC para diseñar y poner en práctica programas de educación continua, desarrollar sistemas administrativos eficientes y orientados al cliente interno y externo, y elevar el impacto de la educación superior en la sociedad y los sectores productivos es un reto que deben asumir las autoridades y el profesorado universitario en el corto plazo.

Por suerte hoy, gracias a los hallazgos de la investigación en el campo de las TIC aplicadas a la educación, conocemos las características de experiencias exitosas, las tendencias actuales de la demanda, las necesidades educativas de la sociedad y las razones de muchos de los fracasos del pasado. Esto nos permite emprender la difícil tarea del cambio educativo con mayor oportunidad de éxito. El resto es voluntad y responsabilidad política, visión y compromiso con la sociedad de quienes administran la educación para que continúe cumpliendo con sus funciones históricas de contribuir al desarrollo y el mejoramiento de la calidad de vida, a través de la generación, la crítica y la transmisión de los bienes más preciados de la humanidad: el conocimiento, el arte y la ciencia. 


\section{Referencias bibliográficas}

Banyard, A., Cassells, P., Green, J., Hartland, N., Hayes y Reddy, P. Introducción a los procesos cognitivos. Barcelona: Ariel, 1995.

Bates, A. W. Cómo gestionar el cambio tecnológico. Estrategias para los responsables de centros universitarios. Barcelona: Gedisa, 2001.

Burke, James y Ornstein, Robert. The Axemaker's gift. Technology's Capture and Control of Our Minds and Culture. New York: Tarcher Penguin, 1997.

Cabero, Julio. Tecnología educativa. Diseño y utilización de medios en la enseñanza. Barcelona: Paidós, 2001.

Cabero, Julio, Martínez, F. y Salinas, J. [Coords.]. Prácticas fundamentales de tecnología educativa. Barcelona: Oikos-tau, 1999.

Castells, Manuel. The Internet Galaxy. Oxford: University Press, 2001.

Días-Barriga, Frida y Hernández, Gerardo. Estrategias docentes para un aprendizaje significativo. Una interpretación constructivista. México: McGrawHill, 2001.

García, Rolando. El conocimiento en construcción. De las formulaciones de Jean Piaget a la teoría de sistemas complejos. España: Gedisa, 2000.

Klikgsberg, Bernardo. Hacia una Economía con Rostro Humano. San José, Costa Rica: Instituto de Altos Estudios, 2004.

Lee, H. Teacher's Perceptions of Technology: Four Categories of Concerns. [Documento WWW]. Recuperado: http://www.eric.ed.gov/

ERICWebPortal/Home.portal? $\underline{\text { nfpb }}=$ true\&Clearme $=$ true $\&$ pageLabel=ERIC_Search 2001. (Consulta noviembre, 2004).

Majò, Joan y Marquès, Pere. La revolución educativa en la era Internet. Barcelona: Praxis, 2000.

McLuhan, Marshall. Understanding Media. The Extensions of Man. Massachusetts: MIT Press, 1994.

McLuhan, Marshall. The Gutenberg Galaxy. Canada: University of Toronto Press, 2000.

Mehlinger, H. y Powers, S. Technology \& Teacher Education. A Guide for Educators and Policymakers. U.S.A.: College Teaching Series, 2000.

Monereo, C. y Pozo, J. I. La universidad ante la nueva cultura educativa. Enseñar y aprender para la autonomía. Madrid: Síntesis, 2003.

Novek, Eleonor. Do professors dream of Electric Sheep? Academic Axiety about the Information Age. [Documento WWW]. Recuperado:

http://www.eric.ed.gov/ ERICWebPortal/Home.portal? nfpb=true\&Clearme $=$ true \& pageLabel=ERIC_Search 1996. (Consulta noviembre, 2004).

Novek, Eleonor. Do Professors Dream Of Electronic Students? Faculty Anxiety And The New Information Technologies. [Documento WWW]. Recuperado: http://www.eric.ed.gov/ ERICWebPortal/Home.portal?

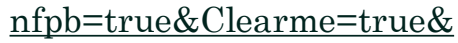
pageLabel=ERIC_Search, 1999. (Consulta noviembre, 2004). 
Olson, David. El mundo sobre el papel. El impacto de la escritura y la lectura en la estructura del conocimiento. España: Gedisa, 1999.

Piaget, Jean. Inteligencia y afectividad. Argentina: Aique, 2001.

Rodríguez, Martín. [Coord.]. Didáctica general. Qué y cómo enseñar en la sociedad de la información. Madrid: Biblioteca Nueva, 2002.

Rosas, Ricardo y Sebastián, Christian. Piaget, Vigotski y Maturana. Constructivismo a tres voces. Argentina: Aique, 2001.

Shelley, B., Wepner and Nancy Ziomek, y Liqing, Tao. Three Teacher Educators' Perspectives about the Shifting Responsibilities of Infusing Technology into the Curriculum. Action in Teacher Education. 24 No 4. 53-63. 2003.

Vygotski, Liev Semionovich. Obras escogidas. Tomo III. Madrid: Visor, 1995.
Young, S. y Shaw, D. (1999). Profiles of Effective College and University Teachers. The Journal of Higher Education, Noviember, December, 70(6), 670-685.

Zabalza, Miguel. La enseñanza universitaria. El escenario y sus protagonistas. Madrid: Narcea, 2002.

Zabalza, Miguel. Competencias docentes del profesorado universitario. Calidad $y$ desarrollo profesional. Madrid: Narcea, 2003.

California Community College. Faculty Instructional Technology Needs Assessments and Survey Results. @ONE Summary Report. Office of the Chancellor, 1998.

Research Institute for Higher Education of Hiroshima University. Higher Education Reform for Quality Higher Education Management in the 21th. Century. Economical, Technological, social and political Forces Affecting Higher Education. Japan: Takatoo Print Media, 2000. 